impact of schizophrenia on various resources in Ireland.

Schizophrenia is a chronic disease associated with a significant and long-lasting health, social, and financial burden, not only for patients but also for families, other caregivers, and the wider society. Knapp et al, in their widely cited systematic review and English study, have pointed out that the inpatient admission is the single largest contributor to the direct costs of treating schizophrenia. ${ }^{2,3}$ Hence I wish to point out a better way of estimating this direct cost of schizophrenia - by using the annual reports published by the Health Research Board (HRB), Dublin, Ireland. ${ }^{4}$

Behan et a/ have used the data about inpatients with schizophrenia, estimated on the recording of inpatient data using the hospital inpatient enquiry system (HIPE), which records the discharge diagnoses using the ICD-10, coding system. The authors' estimation is based on certain assumptions; and hence it is an approximation.

Behan et al found that psychosis was the primary diagnosis in $0.1 \%$ of the total discharges from Irish hospitals in the year 2006. They used the methodology of Mangalore and Knapp's cost-of-illness in schizophrenia in England, which assumes that on an average $40 \%$ of all those who have psychoses will have schizophrenia. ${ }^{3}$ In the cost studies the results are based upon the quality and the accuracy of the patients' databases used.

There is another more reliable and accurate source of data, based on the Activities of the Irish Psychiatric Units and Hospitals, 2006, published by the HRB. Since 1965, all the psychiatric units and hospitals in Ireland, send quarterly returns, information about all psychiatric inpatients, in electronic format, to the HRB national psychiatric inpatients reporting system (NPIRS). These are used by the HRB to publish their annual reports. ${ }^{4}$

According to the HRB report 2006; there were 20,288 admissions to Irish psychiatric units and hospitals. Schizophrenia accounted for $20 \%$ of all and $13 \%$ of first admissions and has consistently remained the second leading cause of psychiatric admissions. Half of all admissions to units and hospitals in 2006 were to general hospital psychiatric units, $32 \%$ were to psychiatric hospitals (including the Central Mental Hospital, Dundrum; St Joseph's Intellectual Disability Service, Portrane; and Carraig Mor, Cork) and 19\% were to private hospitals. The proportion of non-voluntary admissions remained unchanged from 2005 at $11 \%$ of all and $12 \%$ of first admissions. Only 3\% of admissions to private hospitals were non-voluntary. A total of 847 patients with a diagnosis of schizophrenia $(22 \%)$ had non-voluntary admissions.

There were 20,098 discharges from, and 161 deaths in, Irish psychiatric units and hospitals in 2006. Schizophrenia, schizo-typal and delusional disorders accounted for 3,861 discharges (19.4\% of total discharges) and 150,275 inpatient days (bed-days), which accounted for $27.4 \%$ of the total inpatient days, ie. 547,779. In patients with schizophrenia the median length of stay was 21 days, compared to 14 days for all discharges. ${ }^{4}$

Based on this report I have recalculated the bed-days and found that Behan et al have possibly underestimated the direct costs of schizophrenia. I found that inpatients with schizophrenia, admitted to various psychiatric units in general hospitals in Ireland, accounted for approximately 75,000 bed days $(56,279$ Behan et al). While the figures for patients with schizophrenia admitted in psychiatric hospitals were 48,000 $(38,227$ Behan et al) and for private hospitals were 27,000 $(10,167$ Behan et al). This would increase the direct cost by $€ 14-15$ million, making total direct costs approximately $€ 130$ million.

The cost of Mental Health Tribunals should also be included in the estimation. A total 847 patients with schizophrenia had non-voluntary admissions under the Mental Health Act 2001. Even if only 800 had their tribunals, the total cost would be significant.

Ajay Dixit MD, DPM,

Senior House Officer, Old Age Psychiatry, Cork University Hospital, Ireland

References

1. Behan $C$, Kennelly $B, O^{\prime}$ Callaghan $E$. The economic cost of schizophrenia in Ireland: a cost of illness study. Ir J Psych Med 2008; 25(3): 80-87.

2. Knapp M, Mangalore R, Simon J. The Global Costs of Schizophrenia. Schiz Bull 2004; 30(2): 2004.

3. Mangalore R, Knapp M. Cost of schizophrenia in England. J Mental Health Policy 3. Mangalore R, Knapp M. Cost

4. Daly A, Ward M, Moran R, Walsh D. Activities of Irish Psychiatric Units and Hospitals 2006. Health Research Board, annual reports, Dublin 2006. www.hrb.ie

\section{Inpatient co-morbidity of mental illness and substance misuse (including alcohol)}

Dear Editor - Co-morbidity (simultaneous presence of two or more disorders) of mental illness and substance misuse is well established, but unfortunately, poorly identified by the psychiatric services. A significant number of psychiatric patients with substance dependence/misuse co-morbidity receive no specialist intervention.'

Substance misuse co-morbidity has been associated with; increased psychiatric admissions, violence, suicidal behaviour, excess service costs and poor treatment outcome. ${ }^{1}$ Ireland has one of the highest per capita alcohol consumptions in Europe. Ireland also tops the country scale for heavy drinking in general population in Europe..$^{1-3}$

The improved management of co-morbid mental illness and substance misuse is currently a National Health Service (NHS) priority in England and indeed the expert group on mental health policy in Ireland also recommend harvesting this relevant information from patients' databases, in order to optimise the management of co morbidity here in Ireland. ${ }^{1-3}$

Recent European studies of psychiatric patients, reported the prevalence of alcohol misuse in up to $50.6 \%$ of males and $29.2 \%$ of females, and cannabis misuse in $35.2 \%$ of males and $11.2 \%$ of females. ${ }^{1,4,5}$ An Irish study of 50 longstay psychiatric patients living in high support community residences in Dublin has reported the levels of co-morbid alcohol misuse at $48 \%$ and substance misuse as $36 \%{ }^{6}$

An unpublished study, at an inpatient psychiatric unit at the Mercy University Hospital, Cork, found co-morbidity of substance misuse (including alcohol) in over 30\% acute psychiatric admissions.?

All psychiatric units in Ireland, since the year 1965, send information about their inpatients on the national psychiatric inpatients reporting system (NPIRS). This includes information not only on patients' demographics but also both 
